\title{
Unusual Serial Electrocardiographic Changes which Progressed to Arrhythmogenic Right Ventricular Cardiomyopathy
}

\author{
Shu Yoshihara ${ }^{1,2}$, Masaki Matsunaga ${ }^{2}$, Taku Yaegashi $^{3}$, Shioto Suzuki ${ }^{4}$, \\ Masaaki Naito ${ }^{1}$ and Yasuo Takehara ${ }^{5}$
}

\begin{abstract}
Left ventricular (LV) involvement in the advanced stage of arrhythmogenic right ventricular cardiomyopathy (ARVC) is a well recognized phenomenon. $T$ wave inversion in the lateral leads has been reported to be an electrocardiographic marker of LV involvement. Variants of ARVC that preferentially affect the left ventricle (left-dominant subtype of arrhythmogenic cardiomyopathy) have recently been recognized. We herein report a case in which an initial electrocardiogram that was similar to the left-dominant subtype of arrhythmogenic cardiomyopathy progressed to definitive ARVC over a period of 7 years. This case supports the hypothesis that LV involvement in ARVC may precede the evident onset of significant RV dysfunction.
\end{abstract}

Key words: arrhythmogenic right ventricular cardiomyopathy, arrhythmogenic cardiomyopathy, left dominant arrhythmogenic cardiomyopathy

(Intern Med 55: 1103-1108, 2016)

(DOI: 10.2169/internalmedicine.55.5976)

\section{Introduction}

Arrhythmogenic right ventricular cardiomyopathy (ARVC) is an inherited heart muscle disorder characterized by ventricular arrhythmias and sudden cardiac death, especially in the young (1). Pathologically, ARVC is characterized by the degeneration of cardiac myocytes with replacement by fibro-fatty tissue. ARVC predominantly affects the right ventricle, but it can involve the left ventricle. In a multicenter pathological study, left ventricular (LV) involvement occurred with disease progression and it was histologically present in $76 \%$ of all cases (2). The diagnosis of ARVC is based on a comprehensive evaluation of the clinical, imaging, histological, and electrocardiographic findings from the Task Force criteria (3). Among these, the electrocardiogram (ECG) provides important diagnostic clues in patients suspected of having right ventricular (RV) cardiomyopathy.

We herein report a case of ARVC in which serial ECGs demonstrated unusual progressive changes. An initial ECG that had a non-specific $\mathrm{T}$ wave inversion in the lateral and inferior leads unexpectedly developed into definitive ARVC over the course of 7 years.

\section{Case Report}

An asymptomatic 61-year-old man was referred to our hospital because of progressive ECG abnormalities. Seven years prior to his visit, he had presented with abnormal ECG findings for the first time in another health examination center and underwent echocardiography to screen for cardiac pathology, which revealed a reduced LV wall motion in the apex. Thereafter, he was observed by follow-up ECG every 2 years at the same health examination center. He had no medical history of cardiovascular diseases. He also had no family history of cardiomyopathy or sudden death. A physical examination did not show any abnormality. Ophthalmological and dermatological evaluations also did not

\footnotetext{
${ }^{1}$ Department of Diagnostic Radiology, Iwata City Hospital, Japan, ${ }^{2}$ Department of Cardiology, Iwata City Hospital, Japan, ${ }^{3}$ Department of Radiological Technology, Iwata City Hospital, Japan, ${ }^{4}$ Department of Pathology, Iwata City Hospital, Japan and ${ }^{5}$ Department of Radiology, Hamamatsu University School of Medicine, Japan

Received for publication June 12, 2015; Accepted for publication August 6, 2015

Correspondence to Dr. Shu Yoshihara, shuy@hospital.iwata.shizuoka.jp
} 


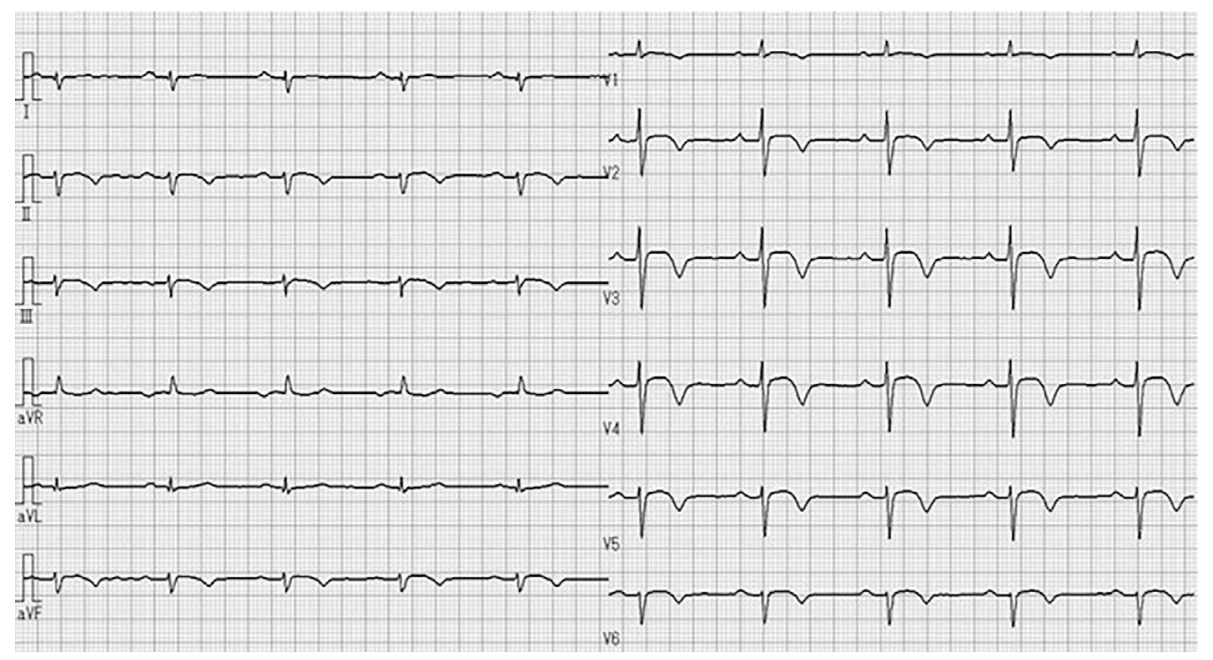

Figure 1. A resting 12-lead electrocardiogram at the first visit to our hospital showed a right ventricular hypertrophy pattern with negative T waves in leads II, III, aVF, and V1 through V6.

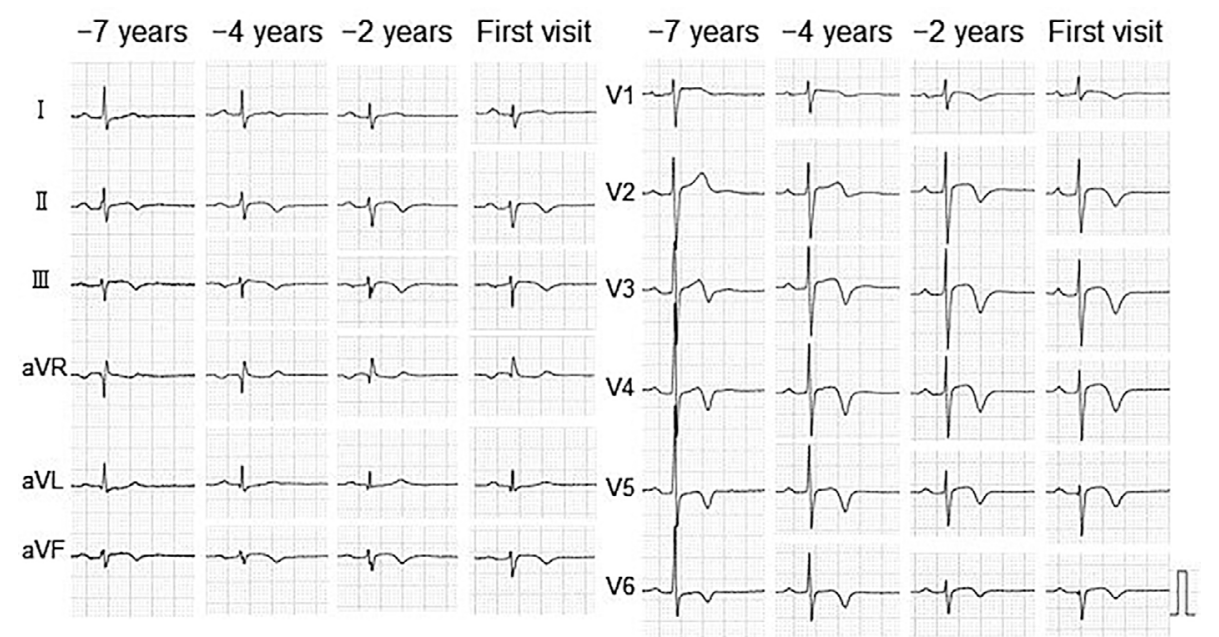

Figure 2. Serial resting 12-lead electrocardiograms (ECGs). Seven years before the first visit to our hospital (-7 years), ECG showed normal QRS voltage and normal $R$ wave progression in the precordial leads. In addition, $T$ wave inversion was limited in the inferior and mid to left precordial leads. Serial ECGs showed gradual attenuation of $S$ wave amplitude in $V 1$ and $R$ wave amplitude in left precordial leads, which resulted in a reversal of the normal $R$ wave progression in the precordial leads. Serial ECGs also demonstrated that $\mathbf{T}$ wave inversion gradually extended to the right precordial leads.

detect abnormal findings. Resting 12-lead ECG at the first visit to our hospital showed a RV hypertrophy pattern with negative $\mathrm{T}$ waves in leads II, III, aVF, and V1 through V6 (Fig. 1). His resting 12-lead ECG obtained 7 years previously showed a normal QRS voltage $(\mathrm{SV} 1=0.78 \mathrm{mV}$, RV5 $=2.06 \mathrm{mV}$ ), and normal $\mathrm{R}$ wave progression in the precordial leads. In addition, $\mathrm{T}$ wave inversion was more limited in the inferior and mid to left precordial leads. Serial ECGs showed a gradual attenuation of the $\mathrm{S}$ wave amplitude in V1 and $\mathrm{R}$ wave amplitude in the left precordial leads, which resulted in a reversal of the normal $\mathrm{R}$ wave progression in the precordial leads. Serial ECGs also indicated a gradual extension of $\mathrm{T}$ wave inversion to the right precordial leads (Fig. 2). Chest radiography was normal with a cardiotho- racic ratio of $50 \%$. Laboratory examinations showed an elevated brain natriuretic peptide level of $96.1 \mathrm{pg} / \mathrm{mL}$ (normal range $\leq 19.5 \mathrm{pg} / \mathrm{mL}$ ). The serum angiotensin-converting enzyme and soluble interleukin-2 receptor level was normal. Chest computed tomography revealed no hilar or mediastinal lymphadenopathy and there was also no abnormal shadow in the lung field. Transthoracic echocardiography showed LV akinesia in the apex with a well preserved overall LV systolic function. The echocardiographic LV end-diastolic diameter and LV ejection fraction (EF) were $47 \mathrm{~mm}$ and $60 \%$, respectively. The RV dimension was mildly increased, measuring $34 \mathrm{~mm}$ at the parasternal long axis view. However, initial echocardiographic examinations could not detect any RV wall motion abnormality. The patient was first re- 


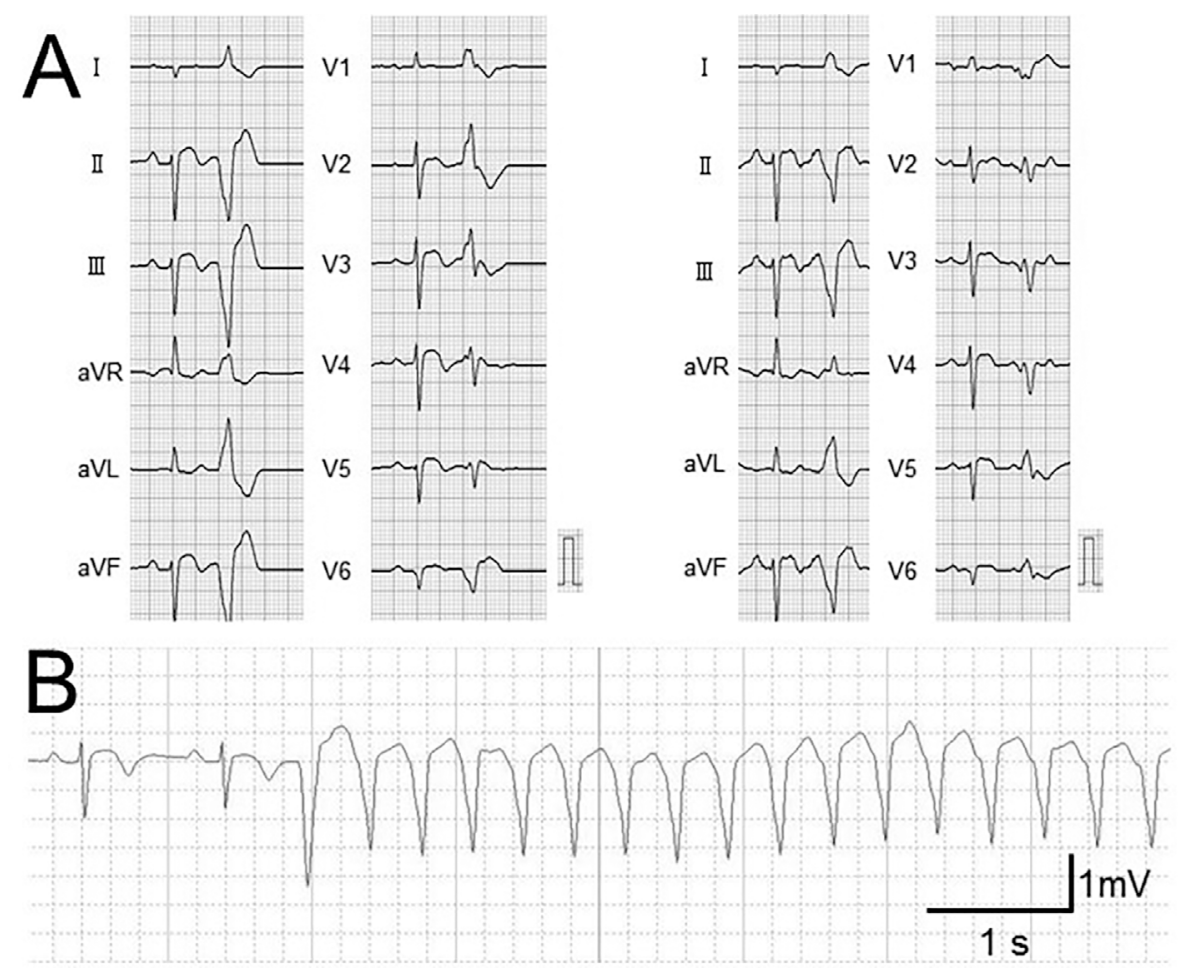

Figure 3. (A) An exercise electrocardiogram (ECG) showing 2 types of premature ventricular contractions that have right bundle branch block morphology with a superior QRS axis (left) and left bundle branch block morphology with a superior QRS axis (right). (B) Non-sustained ventricular tachycardia recorded in the NASA lead during the 24-hour ambulatory ECG.

ferred for exercise stress-rest single-photon emission computed tomography (SPECT) myocardial perfusion imaging and cardiac computed tomography angiography (CTA) to rule out the presence of obstructive atherosclerotic coronary artery disease. The exercise electrocardiographic results were negative for ischemia; however, it showed 2 types of premature ventricular contractions having a right bundle branch block morphology with a superior QRS axis (Fig. 3A, left) and a left bundle branch block morphology with a superior QRS axis (Fig. 3A, right). Technetium-99 $\mathrm{m}$ tetrofosmin SPECT showed a fixed perfusion defect in the apex of the left ventricle (Fig. 4A, arrowhead). Cardiac CTA did not show any critical obstructive coronary artery disease. However, cine-mode CTA revealed regional akinesia of the RV free wall (Fig. 4B, arrows) in addition to the akinetic apical LV wall motion (Fig. 4B, arrowhead). According to the CTA results, cardiac magnetic resonance imaging (MRI) and cardiac catheterization were performed. Cardiac cine MRI also showed regional akinesia of the RV free wall. An analysis of the RV function showed a mildly increased end diastolic volume index of $106 \mathrm{~mL} / \mathrm{m}^{2}$ and reduced $\mathrm{EF}$ of $33 \%$. In addition, cardiac MRI showed the presence of an akinetic area at the apex of the LV and also demonstrated a mid-wall late gadolinium enhancement in the LV apex (Fig. 4C, arrowhead). An analysis of the LV function showed a normal end diastolic volume index of $76 \mathrm{~mL} / \mathrm{m}^{2}$ and mildly diminished $\mathrm{EF}$ of $52.5 \%$. An increase in the RV to $\mathrm{LV}$ end diastolic volume ratio to 1.4 was also observed. Coronary angiogra- phy revealed no significant coronary artery stenosis. RV angiography confirmed the presence of regional akinesia of the $\mathrm{RV}$ free wall. An endomyocardial biopsy was performed from the RV side of the interventricular septum and 3 samples were thus obtained. A histopathological examination showed an extensive loss of the myocardium with fibro-fatty (mainly fibrous) replacement (Fig. 5). Noncaseating epithelioid-cell granuloma and multinucleated giant cells were not detected. 24-hour ambulatory ECG recorded 755 ventricular extrasystoles and 11 episodes of non-sustained ventricular tachycardia (Fig. 3B). Based on these findings, the patient thus satisfied three of the major and one of the minor 2010 revised Task Force criteria and was diagnosed with definite ARVC. Mutation screening for desmosomal genes was refused by the patient and thus not performed. After obtaining adequate informed consent about the risk of sudden death and the future necessity of an implantable cardioverter-defibrillator, he was treated with amiodarone.

\section{Discussion}

A diagnosis of ARVC may often be suspected based on ECG abnormalities. The ECG changes in ARVC reflect the pathophysiology of ARVC, which is characterized by the progressive replacement of myocardial tissue by fibrous and fatty tissue (4). One of the common ECG presentations in ARVC is $\mathrm{T}$ wave inversion on right precordial leads (5). $\mathrm{T}$ wave inversion is often a manifestation of myocardial injury 

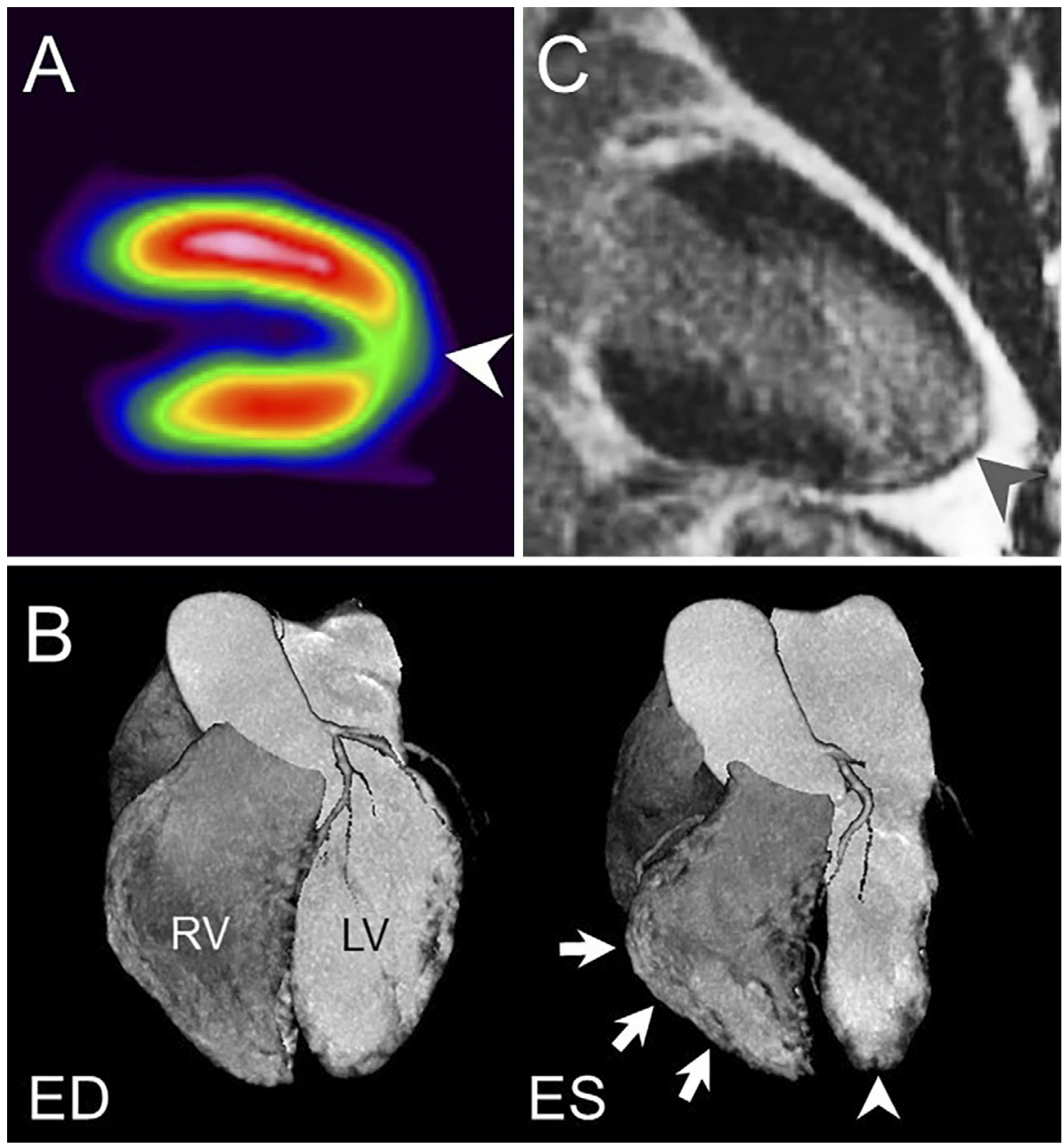

Figure 4. (A) Vertical long axis view of rest technetium-99m tetrofosmin single-photon emission computed tomography myocardial perfusion imaging showing perfusion defect in the apex of the left ventricle (arrowhead). (B) Four-dimensional ventriculography of the cardiac computed tomography angiography showing regional akinesia of the right ventricular free wall (arrows) and akinetic apical left ventricular wall motion (arrowhead). ED: end diastole, ES: end systole, RV: right ventricle, LV: left ventricle. (C) Vertical long axis view of the contrast-enhanced cardiac magnetic resonance image showing mid-wall late gadolinium enhancement in the left ventricular apex (arrowhead).

and it is encountered in a variety of clinical conditions such as ischemic heart disease, cardiomyopathy, and cerebral and pulmonary disorders (6). In ARVC, T wave inversion (repolarization abnormality) in right precordial leads (V1-V3) are included in the 2010 revised Task Force criteria and they constitute a major criterion in the absence of complete right bundle branch block (3). Although ARVC is predominantly a disease of the right ventricle, it is now well established that involvement of the left ventricle may also sometimes occur. Corrado et al. presented a clinical-pathological overview of ARVC in a multicenter study of 42 patients who had suffered arrhythmia- or heart failure-related death or had undergone cardiac transplantation. According to their results, LV involvement occurred with disease progression and was present histologically in $76 \%$ of the cases. In addition, they demonstrated that $\mathrm{T}$ wave inversion in the lateral leads (V5, V6) was an ECG marker of LV involvement (2). A later study clarified that RV disease appeared to be significantly more advanced among ARVC subjects with LV involvement compared with ARVC subjects without LV involvement (7). Recently, the recognition of disease subtypes has broadened the clinical spectrum of ARVC and thus supports the adoption of the broader term arrhythmogenic cardiomyopathy. According to Sen-Chowdhry et al., three patterns of disease expression were identified: (1) classic right-dominant, with isolated RV disease or LV involvement in association with significant RV impairment; (2) left-dominant, with early and prominent LV manifestations and relatively mild right-sided disease; and (3) biventricular, characterized by parallel involvement of both ventricles. The classic right-dominant subtype typically presents an increased RV to LV end diastolic volume ratio exceeding 1.2 on cardiac MRI. In contrast, the RV to LV end diastolic volume ratio remains $<1$ in the left-dominant subtype (7). The left-dominant subtype of arrhythmogenic cardiomyopathy is pathologically characterized by fibro-fatty replacement of the left ventricle, which 


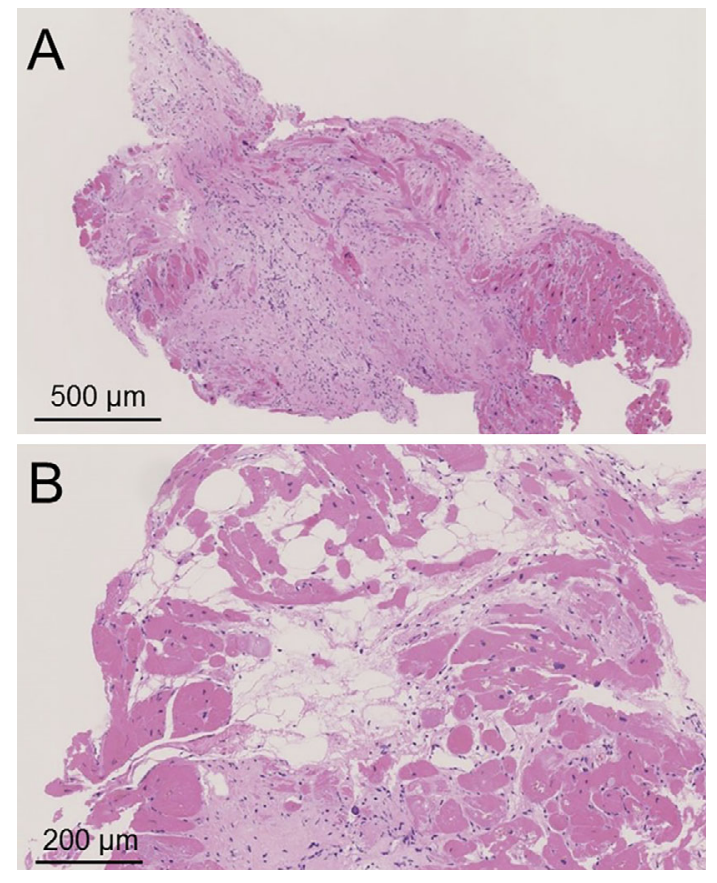

Figure 5. Histological images of the endomyocardial biopsy materials obtained from the right ventricular side of the interventricular septum (Hematoxylin and Eosin staining). The histopathological finding of one of the biopsy samples showed an extensive loss of myocardium (estimated residual myocytes $<50 \%$ ) with fibrous replacement (A). The histopathological finding of another biopsy sample showed fibro-fatty replacement of the myocardium (B).

often occurs as a circumferential band in the outer one-third of the myocardium and the right side of the interventricular septum (8). Relevant clinical features of the left-dominant subtype include inverted $\mathrm{T}$ waves in the lateral and/or inferior leads at ECG and ventricular arrhythmias of right bundle branch block morphology, which are consistent with a LV origin $(8,9)$.

In the case reported herein, we were able to obtain serial ECGs over 7 years. The patient's ECG from 7 years before his first visit showed $\mathrm{T}$ wave inversion localized in lateral and inferior leads. In addition, echocardiography showed reduced LV wall motion in the apex at that time. We could not definitively diagnose our case as having left-dominant subtype arrhythmogenic cardiomyopathy because he had been followed up from another health examination center and we did not have all of the data regarding cardiac MRI or ventricular arrhythmias from the previous 7 years. However, his ECG from 7 years previously was consistent with the left-dominant subtype of arrhythmogenic cardiomyopathy (9). Thereafter, $T$ wave inversion gradually extended to the right precordial leads and an electrocardiographic RV hypertrophy pattern became evident over time. After the passage of 7 years, we were able to make a definitive diagnosis of ARVC according to the 2010 revised Task Force criteria (3). The RV to LV end diastolic volume ratio increased to 1.4 on cardiac MRI, which correlated with the previous findings on the classic right-dominant subtype of arrhythmogenic cardiomyopathy (7). The ECG change in our case is different from the previous findings reported by Jaoude et al. in classic right-dominant subtype arrhythmogenic cardiomyopathy (ARVC) (10). In contrast, SenChowdhry et al. reported the long-term electrocardiographic follow-up over 5 years in 3 patients with left-dominant subtype arrhythmogenic cardiomyopathy (8). Two patients who had $\mathrm{T}$ wave flattening/inversion in V5 to V6 on the initial evaluation gradually extended to V4, V3, V2, and the inferior leads during the follow-up, which is similar to our case. However, they did not show any actual 12-lead ECG tracing. To our knowledge, ours is the first report of ARVC in which serial ECGs actually demonstrate the progressive $\mathrm{T}$ wave inversion from the left precordial to right precordial leads.

In summary, we herein reported a case of ARVC that presented with a unique pattern of serial electrocardiographic change. This case supports the hypothesis that LV involvement in ARVC may precede the evident onset of significant $\mathrm{RV}$ dysfunction. We should therefore be aware that nonspecific $\mathrm{T}$ wave inversion in the lateral and/or inferior leads, which is a common ECG finding in daily clinical practice, may thus be found in the time course of disease progression in ARVC with LV involvement.

The authors state that they have no Conflict of Interest (COI).

\section{References}

1. Calkins H. Arrhythmogenic right ventricular dysplasia/cardiomyopathy: three decades of progress. Circ J 79: 901-913, 2015.

2. Corrado D, Basso C, Thiene G, et al. Spectrum of clinicopathologic manifestations of arrhythmogenic right ventricular cardiomyopathy/dysplasia: a multicenter study. J Am Coll Cardiol 30: 1512-1520, 1997.

3. Marcus FI, McKenna WJ, Sherrill D, et al. Diagnosis of arrhythmogenic right ventricular cardiomyopathy/dysplasia: proposed modification of the task force criteria. Circulation 121: 1533-1541, 2010.

4. Marcus FI, Zareba W. The electrocardiogram in right ventricular cardiomyopathy/dysplasia. How can the electrocardiogram assist in understanding the pathologic and functional changes of the heart in this disease? J Electrocardiol 42: 136.e1-136.e5, 2009.

5. Nasir K, Bomma C, Tandri H, et al. Electrocardiographic features of arrhythmogenic right ventricular dysplasia/cardiomyopathy according to disease severity: a need to broaden diagnostic criteria. Circulation 110: 1527-1534, 2004.

6. Said SA, Bloo R, de Nooijer R, Slootweg A. Cardiac and noncardiac causes of T-wave inversion in the precordial leads in adult subjects: a Dutch case series and review of the literature. World J Cardiol 7: 86-100, 2015.

7. Sen-Chowdhry S, Syrris P, Ward D, Asimaki A, Sevdalis E, McKenna WJ. Clinical and genetic characterization of families with arrhythmogenic right ventricular dysplasia/cardiomyopathy provides novel insights into patterns of disease expression. Circulation 115: 1710-1720, 2007.

8. Sen-Chowdhry S, Syrris P, Prasad SK, et al. Left-dominant arrhythmogenic cardiomyopathy: an under-recognized clinical entity. J Am Coll Cardiol 52: 2175-2187, 2008. 
Intern Med 55: 1103-1108, 2016 DOI: 10.2169/internalmedicine.55.5976

9. Norman M, Simpson M, Mogensen J, et al. Novel mutation in desmoplakin causes arrhythmogenic left ventricular cardiomyopathy. Circulation 112: 636-642, 2005.
10. Jaoude SA, Leclercq JF, Coumel P. Progressive ECG changes in arrhythmogenic right ventricular disease. Evidence for an evolving disease. Eur Heart J 17: 1717-1722, 1996.

(C) 2016 The Japanese Society of Internal Medicine http://www.naika.or.jp/imonline/index.html 